An International Journal of Optimization and Control: Theories \& Applications

ISSN: 2146-0957 eISSN: 2146-5703

Vol.7, No.3, pp.234-239 (2017)

https://doi.org/10.11121/ijocta.01.2017.00489

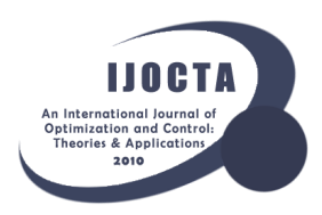

\title{
New function method to the (n+1)-dimensional nonlinear problems
}

\author{
Tolga Akturk ${ }^{a *}$ (D), Yusuf Gurefe ${ }^{b}$, Hasan Bulut $^{c}$ \\ a Department of Mathematics and Science Education, Faculty of Education, Ordu University, Turkey \\ ${ }^{b}$ Department of Econometrics, Faculty of Economics and Administrative Sciences, Usak University, Turkey \\ ${ }^{c}$ Department of Mathematics, Faculty of Science, Firat University, Turkey \\ tolgaakturkk@gmail.com,ygurefe@gmail.com,hbulut@firat.edu.tr
}

\section{ARTICLE INFO}

Article history:

Received: 14 June 2017

Accepted: 30 September 2017

Available Online: 10 October 2017

\section{Keywords:}

New function method

$(N+1)$-dimensional double sine-Gordon

equation

$(N+1)$-dimensional double sinh-cosh-

Gordon equation

Elliptic integral function solutions

AMS Classification 2010:

$35 \mathrm{C} 07,35 \mathrm{CO}, 35 \mathrm{~J} 60$

\section{ABSTRACT}

In this study, a new approach that assumes $u^{\prime}=f(\cos (u))$ and $u^{\prime}=f(\sinh (u))$ is applied to construct the traveling wave solutions of the $(\mathrm{N}+$ 1)-dimensional double sine-Gordon and $(\mathrm{N}+1)$-dimensional double sinh-coshGordon equations. Some new elliptic integral function solutions are respectively obtained by this method, and then these solutions are converted into the Jacobi elliptic function solutions. According these results, one can easily see that this method is very effective mathematical tool for the $(\mathrm{N}+1)$-dimensional nonlinear physical problems.

\section{Introduction}

The nonlinear evolution equations have become of ever greater interest in the modelling of real life problems. Thus, many methods are constructed and applied for these problems. Some of them can be respectively given as the trial equation method [1], the extended trial equation method [2,3], the Weierstrass transform method [4], the tanh function method [5], the Kudryashovs method [6], and so on. In this paper, the investigation of various traveling wave solutions to $(\mathrm{N}+1)$-dimensional double sine-Gordon and $(\mathrm{N}+1)$ dimensional double sinh-cosh-Gordon equations have been widely studied by many authors [7-9]

$$
\begin{aligned}
& \sum_{j=1}^{N} u_{x_{j} x_{j}}-u_{t t}-\alpha \sin (u)-\beta \sin (2 u)=0, \\
& \sum_{j=1}^{N} u_{x_{j} x_{j}}-u_{t t}-\alpha \cosh (u)-\beta \sinh (2 u)=0 .
\end{aligned}
$$

The sine-cosine-Gordon and the sinh-cosh-Gordon equations have importance in the fields of integrable quantum field theory, kink dynamics, and fluid dynamics. On the other hand, a variety of effective methods have been defined to construct the traveling wave solutions of nonlinear partial differential equations. It is given the new function method as one of most important methods and its applications [1014]. In this paper, we apply the new function method, based on sine, sinh functions, to $(\mathrm{N}+1)$-dimensional double sine-Gordon and $(\mathrm{N}+1)$-dimensional double sinh-cosh-Gordon equations. Thus, some new Jacobi elliptic function solutions are obtained by the using of this method. The obtained results reveal that the new function method is powerful mathematical tool for solving the $(\mathrm{N}+1)$-dimensional sine-Gordon and sinhcosh-Gordon equations.

\section{New function method}

The new function methods have been proposed by using the exponential function, trigonometric function $[10,11]$. In this paper, we apply the new function method by depending on the hyperbolic and trigonometric functions. Firstly, we take the general form of the generalized $(\mathrm{N}+1)$-dimensional sinecosine-Gordon or sinh-cosh-Gordon equations,

$$
P\left(\begin{array}{l}
u, \sin (u), \cosh (u), \sin (2 u), \\
, \sinh (2 u) u_{t t}, u_{x_{1} x_{1}}, u_{x_{2} x_{2}}, \ldots u_{x_{N} x_{N}}
\end{array}\right)=0 .
$$

\footnotetext{
*Corresponding author
} 
Then use the wave transformation $u=u(x, t)=u(\mu)=u\left(k\left(\sum_{j=1}^{N} x_{j}-c t\right)\right)$, $\mu=k\left(\sum_{j=1}^{N} x_{j}-c t\right)$

where $c \neq 0$. Thus, we have a nonlinear ordinary differential equation

$$
N\left(u, u^{\prime}, u^{\prime \prime}, \ldots\right)=0 .
$$

The new function method assumes that the function $u$ provides

$$
F\left(u^{\prime \prime}\right)=G(g(u)),
$$

where $F, G$ and $g$ are any functions. Here, we use the equations

$$
\begin{array}{r}
u^{\prime}=f(g(u)), \\
u^{\prime \prime}=f(g(u)) g^{\prime}(u) f^{\prime}(g(u)) .
\end{array}
$$

Substituting Eq. (6) into Eq. (5), we have

$$
F\left(f(g(u)) \mathrm{g}^{\prime}(u) f^{\prime}(g(u))\right)=G(g(u)) \text {. }
$$

If we take $\psi=g(u)$, then we can write

$$
F\left(\psi^{\prime} f(\psi) f^{\prime}(\psi)\right)=G(\psi) .
$$

Solving Eq. (8), is sometimes a variable separated ordinary differential equation, yields the function $f$. By integration, we can obtain the solutions as follows:

$$
\frac{d u}{f(g(u))}=d \mu \Rightarrow \int \frac{d u}{f(g(u))}=\int d \mu=\mu+P \text {, }
$$

where $P$ is an integration constant. The explicit solutions can be derived by the inverse function. Otherwise, the implicit solutions can be retrieved if the above integration is much complex.

\section{Applications}

\subsection{Solutions for $(\mathrm{N}+1)$-dimensional double sine- gordon equation}

By the travelling wave transformation to Eq. (1), we find

$$
k^{2}\left(N-c^{2}\right) u^{\prime \prime}-\alpha \sin (u)-\beta \sin (2 u)=0 .
$$

We assume that the equation

$$
u^{\prime}=f(\cos (u)),
$$

defined by $u^{\prime}(\mu)$ and $\cos (u)$ satisfies Eq. (10) From Eq. (11), we can write

$$
u^{\prime \prime}=-\sin (u) f(\cos (u)) f^{\prime}(\cos (u)) \text {. }
$$

By substituting Eq. (12) into Eq. (10), we derive

$$
\begin{aligned}
& k^{2}\left(c^{2}-N\right) f(\cos (u)) f^{\prime}(\cos (u))= \\
& =\alpha+2 \beta \cos (u)
\end{aligned}
$$

Let $u^{\prime}$ is a function of $\cos (u)$ and $\cos (u)=\psi$, then $f$ is a function of $\psi$. Therefore we can easily write

$$
\begin{aligned}
& u=\arccos ((\psi)) \\
& u^{\prime}=-\frac{\psi^{\prime}}{\sqrt{1-\psi^{2}}}=f(\cos (u))=f(\psi)
\end{aligned}
$$

Now, we can try to have the form of the function $f$ :

$$
k^{2}\left(c^{2}-N\right) f(\psi) f^{\prime}(\psi)=\alpha+2 \beta \psi .
$$

Eq. (15) is an ordinary differential equation of variable separated:

$$
\frac{k^{2}\left(c^{2}-N\right)}{2} f^{2}(\psi)=\alpha \psi+\beta \psi^{2}+P,
$$

where $P$ is a constant of integration. From Eq. (16), we can easily compute

$$
f(\psi)=-\sqrt{\frac{2}{k^{2}\left(c^{2}-N\right)}\left(\alpha \psi+\beta \psi^{2}+P\right)} .
$$

Using the equation $-\frac{\psi^{\prime}}{\sqrt{1-\psi^{2}}}=f(\psi)$, we have

$\psi^{\prime}=\frac{d \psi}{d \mu}=$

$=-\sqrt{\frac{2}{k^{2}\left(c^{2}-N\right)}\left(1-\psi^{2}\right)\left(\alpha \psi+\beta \psi^{2}+P\right)}$

By using of the symbolic computation software program Mathematica, Eq. (18) that is a variable separated ordinary differential equation is solved.

So, the following elliptic integral function $F$ solution to Eq. (1) is obtained as

$\mu+Q=-\sqrt{\frac{2 k^{2}\left(c^{2}-N\right)}{\beta\left(\psi_{2}-\psi_{3}\right)\left(\psi_{1}-\psi_{4}\right)}}$

EllipticF $\left[\begin{array}{l}\arcsin \left(\sqrt{\frac{\left(\psi-\psi_{2}\right)\left(\psi_{1}-\psi_{4}\right)}{\left(\psi-\psi_{1}\right)\left(\psi_{2}-\psi_{4}\right)}}\right), \\ \frac{\left(\psi_{1}-\psi_{3}\right)\left(\psi_{2}-\psi_{4}\right)}{\left(\psi_{2}-\psi_{3}\right)\left(\psi_{1}-\psi_{4}\right)}\end{array}\right]$,

where $Q$ is a constant of integration. $\psi_{i}(i=1, \cdots, 4)$ are roots of equation 


$$
\begin{gathered}
\left(\alpha \psi+\beta \psi^{2}+P\right)\left(1-\psi^{2}\right)=0 \\
\psi_{1}=-1, \quad \psi_{2}=1, \\
\psi_{3}=\frac{-\alpha-\sqrt{\alpha^{2}-4 P \beta}}{2 \beta} . \\
\psi_{4}=\frac{-\alpha+\sqrt{\alpha^{2}-4 P \beta}}{2 \beta}
\end{gathered}
$$

Then, we find

$$
\psi=\frac{\psi_{2}\left(\psi_{1}-\psi_{4}\right)-\psi_{1}\left(\psi_{2}-\psi_{4}\right) \operatorname{sn}^{2}\left[\varphi, \ell^{2}\right]}{\psi_{1}-\psi_{4}-\left(\psi_{2}-\psi_{4}\right) \operatorname{sn}^{2}\left[\varphi, \ell^{2}\right]} .
$$

Replace $\quad \psi \quad$ with $\cos u, \mu \quad$ with $\mu=k\left(\sum_{j=1}^{N} x_{j}-c t\right)$ in (21), and then the explicit solutions for Eq. (1) can be obtained as follows:

$$
u=\arccos \left[\frac{\psi_{2}\left(\psi_{1}-\psi_{4}\right)-\psi_{1}\left(\psi_{2}-\psi_{4}\right) \operatorname{sn}^{2}\left(\varphi, \ell^{2}\right)}{\psi_{1}-\psi_{4}-\left(\psi_{2}-\psi_{4}\right) \operatorname{sn}^{2}\left(\varphi, \ell^{2}\right)}\right],
$$

where,

$$
\begin{aligned}
& \varphi=-\sqrt{\frac{\beta\left(\psi_{1}-\psi_{4}\right)\left(\psi_{2}-\psi_{3}\right)}{2 k^{2}\left(c^{2}-N\right)}\left(\tau\left(\sum_{j=1}^{N} x_{j}-c t\right)+Q\right)} \\
& \text { and } \ell^{2}=\frac{\left(\psi_{1}-\psi_{3}\right)\left(\psi_{2}-\psi_{4}\right)}{\left(\psi_{2}-\psi_{3}\right)\left(\psi_{1}-\psi_{4}\right)}
\end{aligned}
$$

\subsection{Solutions for $(\mathrm{N}+1)$-dimensional sinh-cosh- gordon equation} we get

By the travelling wave transformation to Eq. (2),

$$
k^{2}\left(N-c^{2}\right) u^{\prime \prime}-\alpha \cosh (u)-\beta \sinh (2 u)=0 .
$$

We assume that the equation

$$
u^{\prime}=f(\sinh (u))
$$

defined by $u^{\prime}(\mu)$ and $\sinh (u)$ satisfies Eq. (23). From Eq. (24), we can compute

$$
u^{\prime \prime}=(\cosh (u)) f(\sinh (u)) f^{\prime}(\sinh (u))
$$

By substituting Eq. (25) into Eq. (23), we derive

$$
\begin{aligned}
& k^{2}\left(N-c^{2}\right) f(\sinh (u)) f^{\prime}(\sinh (u))= \\
& =\alpha+2 \beta \sinh (u) .
\end{aligned}
$$

Let $u^{\prime}$ is a function of $\sinh (u)$ and $\sinh (u)=\psi$, then $f$ is a function of $\psi$. Therefore we can easily write

$$
\begin{aligned}
& u=\operatorname{arcsinh}((\psi)) \\
& u^{\prime}=\frac{\psi^{\prime}}{\sqrt{1+\psi^{2}}}=f(\sinh (u))=f(\psi)
\end{aligned}
$$

Now, we can try to have the form of the function $f$ :

$$
k^{2}\left(N-c^{2}\right) f(\psi) f^{\prime}(\psi)=\alpha+2 \beta \psi \text {. }
$$

Eq. (27) is an ordinary differential equation of variable separated:

$$
\frac{k^{2}\left(N-c^{2}\right)}{2} f^{2}(\psi)=\alpha \psi+\beta \psi^{2}+P,
$$

where $P$ is a constant of integration. From Eq. (28), we can easily compute

$$
f(\psi)= \pm \sqrt{\frac{2}{k^{2}\left(N-c^{2}\right)}\left(\alpha \psi+\beta \psi^{2}+P\right)} .
$$

Using the equation $\frac{\psi^{\prime}}{\sqrt{1+\psi^{2}}}=f(\psi)$, we have $\psi^{\prime}=\frac{d \psi}{d \mu}=$

$= \pm \sqrt{\frac{2}{k^{2}\left(N-c^{2}\right)}\left(1+\psi^{2}\right)\left(\alpha \psi+\beta \psi^{2}+P\right)}$

By using of the symbolic computation software program Mathematica, Eq. (30) that is a variable separated ordinary differential equation is solved. So, the following elliptic integral function $F$ solution to Eq. (2) is obtained as

$$
\begin{aligned}
& \mu+Q= \\
& =\frac{\text { EllipticF }\left[\arcsin \left(\sqrt{\frac{\left(\psi-\psi_{2}\right)\left(\psi_{1}-\psi_{4}\right)}{\left(\psi-\psi_{1}\right)\left(\psi_{2}-\psi_{4}\right)}}\right), \ell^{2}\right]}{\varphi},
\end{aligned}
$$

where $Q$ is a constant of integration. $\psi_{i}(i=1, \cdots, 4) \quad$ are roots of equation $\left(\alpha \psi+\beta \psi^{2}+P\right)\left(1+\psi^{2}\right)=0$

$$
\begin{aligned}
& \psi_{1}=-i, \quad \psi_{2}=i, \\
& \psi_{3}=\frac{-\alpha-\sqrt{\alpha^{2}-4 P \beta}}{2 \beta}, \\
& \psi_{4}=\frac{-\alpha+\sqrt{\alpha^{2}-4 P \beta}}{2 \beta}
\end{aligned}
$$


$\psi=\frac{\psi_{2}\left(\psi_{1}-\psi_{4}\right)-\psi_{1}\left(\psi_{2}-\psi_{4}\right) \operatorname{sn}^{2}\left[\varphi, \ell^{2}\right]}{\psi_{1}-\psi_{4}-\left(\psi_{2}-\psi_{4}\right) \operatorname{sn}^{2}\left[\varphi, \ell^{2}\right]}$

Replace $\quad \psi \quad$ with $\sinh u, \mu \quad$ with $\mu=k\left(\sum_{j=1}^{N} x_{j}-c t\right)$ in (33), and then the explicit solutions for Eq. (2) can be obtained as follows:

$$
u=\operatorname{arcsinh}\left[\frac{\psi_{2}\left(\psi_{1}-\psi_{4}\right)-\psi_{1}\left(\psi_{2}-\psi_{4}\right) \operatorname{sn}^{2}\left(\varphi, \ell^{2}\right)}{\psi_{1}-\psi_{4}-\left(\psi_{2}-\psi_{4}\right) \operatorname{sn}^{2}\left(\varphi, \ell^{2}\right)}\right],
$$

where,

$\varphi=-\sqrt{\frac{\beta\left(\psi_{1}-\psi_{4}\right)\left(\psi_{2}-\psi_{3}\right)}{2 k^{2}\left(c^{2}-N\right)}}\left(\tau\left(\sum_{j=1}^{N} x_{j}-c t\right)+Q\right)$ and $\ell^{2}=\frac{\left(\psi_{1}-\psi_{3}\right)\left(\psi_{2}-\psi_{4}\right)}{\left(\psi_{2}-\psi_{3}\right)\left(\psi_{1}-\psi_{4}\right)}$.

\section{2D and 3D graphics of solution}

\subsection{D graphic of solution}

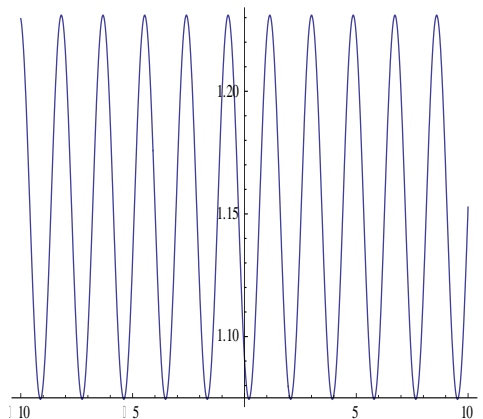

Figure 1. 2D graphic represents the solution (22) at $t=1$.

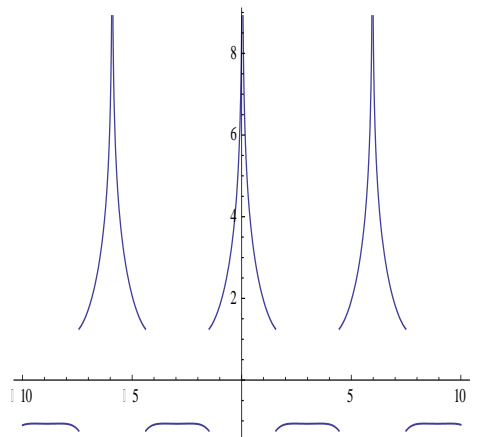

Figure 2. The solution (35) is shown real part at $t=1$.

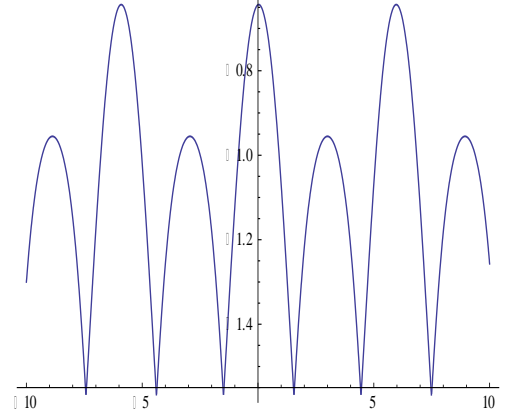

Figure 3. The solution (35) is shown imaginary part at $t=1$.

\subsection{D graphic of solution}

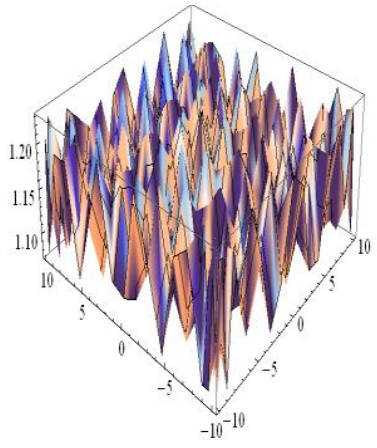

Figure 4. The solution (22) is shown at $\alpha=4, P=0$, $\beta=3, N=1, Q=0, c=3$, and $k=1$

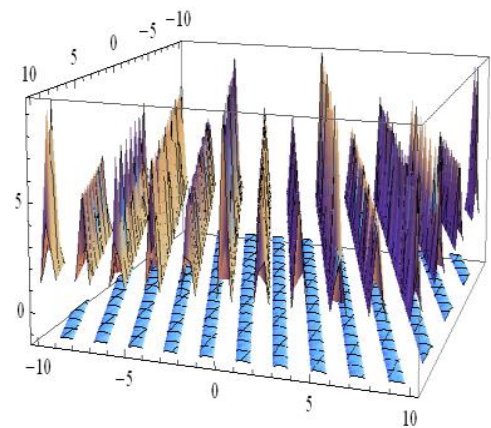

Figure 5. The solution (35) is shown real part at $\alpha=6$, $P=1, \beta=8, n=1, Q=0, c=1$, and $k=3$

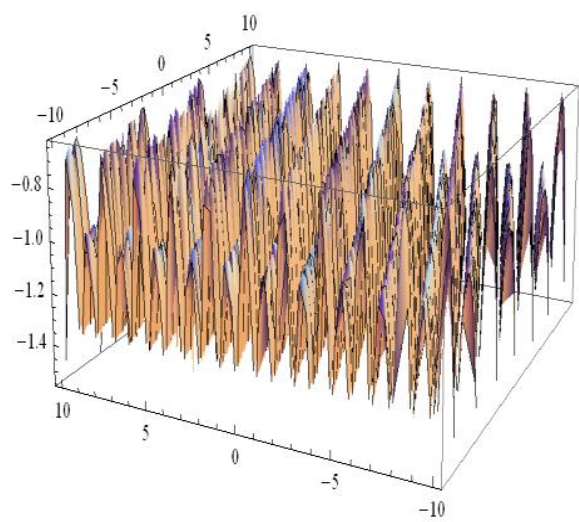

Figure 6. The solution (35) is shown imaginary part at $\alpha=6, P=1, \beta=8, n=1, Q=0, c=1$, and $k=3$ 


\section{Conclusion}

We consider the $(\mathrm{N}+1)$-dimensional double sineGordon and sinh-cosh-Gordon equations to construct new traveling wave solutions by using of the new function method. By these applications, we get some new elliptic integral function solutions. Using simple mathematical transformations, we obtain some new exact solutions based on the Jacobi elliptic function $s n$. The obtained results show that the new function method is very effective mathematical tool for solving the $(\mathrm{N}+1)$-dimensional nonlinear evolution equations.

\section{Acknowledgments}

We would like to thank the reviewers and editors who participated in the evaluation process of our production.

\section{References}

[1] Liu, C. S., (2005). Trial equation method and its applications to nonlinear evolution equations, Acta. Phys. Sin. 54, 2505-2509

[2] Pandir, Y., Gurefe, Y., Kadak, U., \& Misirli, E., (2012). Classification of exact solutions for some nonlinear partial differential equations with generalized evolution, Abstr. Appl. Anal. 2012, 16 pages

[3] Pandir, Y., Gurefe, Y., \& Misirli, E., (2013). Classification of exact solutions to the generalized Kadomtsev- Petviashvili equation, Phys. Scr. 87, 12 pages

[4] Porubov, A.V., \& Velarde, M.G., (1999). Exact periodic solutions of the complex Ginzburg-Landau equation, J. Math. Phys. 40(2), 884-896

[5] Fan, E., (2000). Extended tanh-function method and its applications to nonlinear equations, Phys. Lett. A 277(4), 212-218

[6] Kudryashov, N. A., (2012). One method for finding exact solutions of nonlinear differential equations, Commun. Nonl. Sci. Numer. Simul. 17, $2248-2253$

[7] Li, J.B., (2007). Exact traveling wave solutions and dynamical behavior for the $(n+1)$-dimensional multiple sine-Gordon equation, Sci. in China Ser. A: Math. 50(2), 153-164

[8] Lee, J., \& Sakhtivel, R., (2010). Travelling wave solutions for $(\mathrm{N}+1)$-dimensional nonlinear evolution equations, Pramana-J. Phys. 75(4), 565578
[9] Wang, D.S., Yan Z., \& Li H., (2008). Some special types of solutions of a class of the $(\mathrm{N}+1)$ dimensional nonlinear wave equation, Comput. Math. Appl. 56(6), 1569-1579

[10] Shen, G., Sun, Y., \& Xiong, Y., (2013). New travelling-wave solutions for Dodd-Bullough equation, J. Appl. Math. 2013, Article ID.364718, 5 pages

[11] Sun, Y., (2014). New travelling wave solutions for Sine-Gordon equation, J. Appl. Math. 2014, Article ID.841416, 4 pages

[12] Bulut, H., Akturk, T., \& Gurefe, Y., (2014). Traveling wave solutions of the $(\mathrm{N}+1)$-dimensional sin-cosine-Gordon equation, AIP Conference Proceedings, 1637(1), 145-149

[13] Bulut, H., Akturk, T., \& Gurefe, Y., (2015). An application of the new function method to the generalized double sinh-Gordon equation, AIP Conference Proceedings, 1648(370014), 4 pages

[14] Akturk, T., (2015). Determining the exact solutions of some nonlinear partial differential equations by trial equation methods, Firat University, PhD Thesis

Tolga Akturk is an assistant professor in Department of Mathematics and Science Education, at Ordu University, Ordu (Turkey). He obtained his M.Sc. degree from Firat University and Ph.D. degree from Firat University. His areas of interest are numerical solutions of the linear or nonlinear partial differential equations.

Yusuf Gurefe is an assistant professor in Department of Econometrics, at Usak University, Usak (Turkey). He obtained his M.Sc. degree from Ege University and Ph.D. degree from Ege University. His research interests include multiplicative calculus, analytical and numerical solutions of the linear or nonlinear partial differential equations, nonlinear sciences, mathematical physics.

Hasan Bulut is currently professor of Mathematics in Firat University. His research interests include stochastic differential equations, fluid and heat mechanics, finite element method, analytical methods for nonlinear differential equations and numerical solutions of the partial differential equations. 
An International Journal of Optimization and Control: Theories \& Applications (http://ijocta.balikesir.edu.tr)

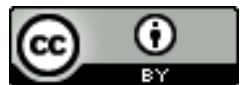

This work is licensed under a Creative Commons Attribution 4.0 International License. The authors retain ownership of the copyright for their article, but they allow anyone to download, reuse, reprint, modify, distribute, and/or copy articles in IJOCTA, so long as the original authors and source are credited. To see the complete license contents, please visit http://creativecommons.org/licenses/by/4.0/. 